\title{
"Un no sé qué que quedan balbuciendo" o los límites de la traducción'
}

\author{
Antonio José Mialdea BaEna \\ Universidad de Córdoba
}

\begin{abstract}
Resumen: La mejor traducción de un verso no necesariamente es la que más se acerca por lengua o cultura al original. En las páginas que siguen proponemos cuatro traducciones de un verso de San Juan de la Cruz perteneciente al Cántico Espiritual y comprobaremos que la traducción alemana respeta tanto el sentido del poeta como los efectos comunicativos que el verso provoca en el lector.
\end{abstract}

\begin{abstract}
The best translation of a line is not necessarily that which is closer to the source language or culture. In this paper we approach four translation of a line by San Juan de la Cruz, included in Cántico Espiritual. We attempt to prove that the German translation respects not only the sense given by the poet but also the communicative effect that the line raises in the reader.
\end{abstract}

Palabras clave: San Juan de la Cruz. Cántico Espiritual.Traducción.

Key words: San Juan de la Cruz. Cántico Espiritual. Translation.

Si la traducción de cualquier texto de una lengua de partida a otra cualquiera de llegada presenta, de suyo, dificultades por numerosas razones, la traducción de textos poéticos se complica, aún más, debido fundamentalmente al proceso de condensación verbal que tiene lugar en la poesía. Por otra parte, hay versos cuya traducción a otras lenguas cobra, si cabe, un grado de dificultad mayor porque en ellos no sólo se trata de trasladar las palabras a sus correspondientes en otros idiomas, como una simple cuestión de analogía, sino que la complicación más grave proviene del aspecto más externo de la palabra, del lenguaje mismo. Por tanto, la forma (en la que entra la grafia, estructura métrica, sonido...) de un verso puede llegar a constituir su sentido más profundo. Algo semejante expresa

\footnotetext{
' Agradezco a los profesores Garcia Peinado y López Folgado sus consejos sobre traducción literaria y, de igual forma, agradezco también a los carmelitas P. Antonio Ángel Sánchez y P. José Fernández Marin sus rápidas diligencias para disponer de las distintas traducciones de los versos sanjuanistas.
} 
Newmark cuando afirma que "there is an emphasis on the form as well as the content of the message", .

En conclusión, es necesario atender a un buen número de aspectos para realizar una buena traducción poética Redundando en este aspecto, Valentín Garcia Yebra ha llegado a afirmar que:

al traductor literario, en la tarea expresiva, se le exige, en cierto modo, más que al autor original. El autor puede añadir o quitar, sustituir o alterar, elementos expresivos, y aun estructurales, sin que la obra sufra por ello; es incluso normal que mejore con las alteraciones. El traductor, en cambio, ha de atenerse con rigor al original; tiene que expresar, en lo posible, todo y solo su contenido, sin concederse más libertades que las estrictamente necesarias (que, por ser necesarias, dejan de ser libertades) ${ }^{3}$

Aparte de esto, el trabajo de traducir se vuelve aún más complicado si de lo que se trata es de traducir poesía mística, donde media una experiencia que los investigadores coinciden en denominar "inefable" y en la que el lenguaje resulta decididamente insuficiente ${ }^{4}$.

En las páginas que siguen nos detendremos en las traducciones inglesa, francesa, italiana y alemana del último endecasílabo de la séptima lira del Cántico Espiritual de San Juan de la Cruz, quizás uno de los versos más bellos y expresivos de la literatura española:

\section{un no sé qué que quedan balbuciendo}

Trataremos de mostrar que la mejor traducción de un verso no siempre y forzosamente tiene que ser la más cercana en cuanto a lengua y cultura.

\section{El verso y su hermenéutica}

Como ya hemos mencionado, se trata del último endecasílabo de la lira séptima del Cántico Espiritual, escrito en la prisión de Toledo entre diciembre de 1577 y enero de 1578, mientras Juan de la Cruz cumple condena, entre otros

\footnotetext{
${ }^{2}$ P. Newmark, A textbook of translation, Hertfordshire: Phoenix ELT, 1995, p. 162 (existe una version española en Madrid, Cátedra, 1999).

"V. García Yebra, "Aurelio Espinosa Polit traductor de poetas clásicos", en Arbor (julio-agosto de 1962), p. 64.

"Cfr. Guillén, Jorge, "Lenguaje insuficiente: San Juan de la Cruz o lo inefable místico" en Lenguaje y poesia. Algunos casos españoles. Revista de Occidente, Madrid, 1962, pp. 95-142. Años más tarde, también A. Ruffinato, "Los códigos del eros y del miedo en San Juan de la Cruz", en Dispositio, University of Michigan, IV (10) 1979, pp., 1-26.

${ }^{5}$ Para el texto sanjuanista seguimos la edición del Cántico Espiritual y poesia completa preparada por Paola Elia y Maria Jesús Mancho, Barcelona, Critica, 2002.
} 
motivos, por ser la cabeza visible de la reforma masculina iniciada años antes por Teresa de Jesús. El contexto poético de la estrofa es el siguiente: el sujeto del poema, que después de caer en la cuenta de la ausencia absoluta de su Amado, ha salido en su busca, inicia una serie de interrogatorios a diferentes entes a quienes pregunta si lo han visto por alguna parte. En la lira séptima Juan de la Cruz redacta la conclusión de esta primera e infructuosa búsqueda:

$$
\begin{gathered}
\text { Y todos cuantos vagan } \\
\text { de ti me van mil gracias refiriendo } \\
y \text { todos más me llagan } \\
\text { y déjame muriendo } \\
\text { un no sé qué que quedan balbuciendo }
\end{gathered}
$$

El mismo Juan de la Cruz la comenta de esta forma ${ }^{7}$ :

"Esto creo no lo acabará bien de entender el que no lo hubiere experimentado; pero el alma que lo experimenta, como ve que se le queda por entender aquello de que altamente siente, llámalo "un no sé qué"; porque, así como no se entiende, así tampoco se sabe decir, aunque, como he dicho, se sabe sentir. Por eso dice que le "quedan" las criaturas "balbuciendo", porque no lo acaban de dar a entender; que eso quiere decir "balbucir", que es el hablar de los niños, que es no acertar a decir y dar a entender qué hay que decir"

Advertimos enseguida que el propio Juan de la Cruz ya nos pone en antecedentes sobre una primera dificultad que cualquier traductor se puede encontrar en el momento de trasladar a otra lengua el verso sanjuanista: la experiencia. Quien no haya experimentado las mismas sensaciones que el autor tiene bastante complicado entender lo que éste quiere expresar. Si ya resulta en extremo difícil trasladar la experiencia, como anteriormente señalamos con el concepto "inefabilidad", a un primer código lingüístico, la lengua madre del escritor, podemos pensar que la dificultad se acrecentará cuando de esta primera lengua la trasladamos a otras distintas.

\footnotetext{
"Esta lira conserva el mismo lugar tanto en la primera como en la segunda redacción que San Juan de la Cruz realizó del Cántico Espiritual.

${ }^{7}$ Recordemos que San Juan de la Cruz escribió un comentario en prosa para cada una de las estrofas del Cántico Espiritual.
} 


\section{Análisis formal del verso}

Pasemos a continuación a realizar un análisis más formal del verso. Lo primero que tenemos que considerar es que, a diferencia de otros versos sanjuanistas, éste no necesita una modernización lingüística, es decir, cualquier hablante actual del español entiende perfectamente cada una de las palabras que en él se contienen. Es posible que tuviésemos que explicar a alguien el significado de 'balbucir' (o 'balbucear') que, según el diccionario de la Real Academia", consiste en "hablar o leer con pronunciación dificultosa, tarda y vacilante, trastocando a veces las letras o las sílabas". Aparte de esta salvedad, el resto del verso es fácilmente comprensible. El hecho de que el verbo principal del verso (balbucir) se encuentre en su forma impersonal de gerundio indica, lógicamente, que la acción posee un continuum que se prolonga sin un final determinado o preestablecido.

Para completar la estructura oracional del verso tenemos que acudir al verso anterior:

\section{$Y$ déjame muriendo \\ Un no sé qué que quedan balbuciendo}

Al pasar de la forma poética a la forma en prosa, tenemos:

Un "no sé que", que (ellos) quedan balbuciendo, me deja muriendo

Se trata, pues, de una oración compuesta de dos proposiciones. La principal, "un no sé qué(algo=indeterminado) me deja muriendo", y una sustantiva de objeto directo, "ellos quedan balbuciéndo(lo)'. Si tuviéramos que decidir cuál es el elemento deteminante del verso diríamos, sin duda, que se encuentra en la porción versal que va desde el 'no' hasta el 'que..'(dan), es decir:

$$
\text { 'no sé qué que que'(dan) }{ }^{10}
$$

La aliteración del fonema oclusivo velar sordo $/ \mathrm{k} /$ en el inicio de palabra produce, por una parte, un efecto sonoro que representa el balbuceo o tartamudeo y que difícilmente reconocerán los hablantes de otras lenguas que no sea la

\footnotetext{
${ }^{8} 21^{3}$ edición, 2001.

'La expresión 'no sé qué' la trataremos como un todo, como un sintagma equivalente a 'algo'.

${ }^{10}$ D. Alonso, al estudiar este verso sanjuanista, escribió: "En general, la sucesión inmediata de tres silabas que resultaria molesta al oido. En este caso, tras la vaguedad del un no sé qué, esa repetición indica una duda, un entrecortado titubeo, que va a complementarse, a recibir su justificación con el gerundio balbuciendo, en el que cuaja la acción verbal" (en Poesia española. Ensayo de métodos y limites estilisticos, Madrid: Gredos, $1966^{5}$, pp. 281).
} 
española (es la función "poética-fónica" a la que se refería Jakobson); y, por otra parte, provoca en el lector una ruptura absoluta del lenguaje, como si la lengua quedara en suspenso y fuera incapaz de decir lo inefable por lo que, de esta forma, tendría que torcer la gramática, la tensión de la lógica, con la suprema inestabilidad del "no sé qué que quedan balbuciendo"11. Proponemos, a continuación, las cuatro versiones citadas.

\section{Cuatro ejemplos de traducción}

De las numerosas lenguas a las que ha sido traducida la obra literaria de San Juan de la Cruz, hemos escogido cuatro de ellas para mostrar el quehacer traductológico respecto del verso que hemos seleccionado: las traducciones italiana $^{12}$, francesa ${ }^{13}$, inglesa ${ }^{14}$ y alemana ${ }^{15}$. Veamos esquemáticamente el resultado de los traslados:

\begin{tabular}{|l|l|}
\hline Italiano & un no so che che dicon balbettando ${ }^{16}$ \\
\hline Francés & un ne sais quoi qu'ils restent balbutiant \\
\hline Inglés & Of, ah, I-don't-know-what behind their stammering \\
\hline Alemán & Das sie nur mühsam stammelnd wiedergeben \\
\hline
\end{tabular}

No hace falta señalar, porque es obvio, que, en el caso que analizamos, las traducciones italiana y francesa se asemejan más a la lengua original del verso que las traducciones inglesa y alemana. Lógicamente, las raíces latinas encontramos de las lenguas española, italiana y francesa difieren de las raíces de las lenguas inglesa y alemana que aquí encontramos. A continuación realizamos una traducción literal al español de los cuatro versos anteriores:

\footnotetext{
"E. Sáinz cita como modelo de la tradición espanoola de lenguaje insuficiente este verso de San Juan de la Cruz en "la poesia pura en Cuba: algunas reflexiones", en Ensayos criticos, Ciudad de la Habana, ed. Unión, 1990, pp. 111-13.

12 La traducción italiana la tomamos de $S$. Giovanni della Croce, opera versione dal $\mathrm{P}$. Ferdinando di S.Maria OCD, Roma, Postulazione Generale dei Juan de la Cruzni Scalzi, 1963.

13 La traducción francesa está tomada de Jean de la Croix. Poésies, traduction de Benoît Lavaud: introductión, chronologie, bibliographie et notes de Bernard Sesé, Paris, GF-Flammarion, 1993.

${ }^{1+}$ El traslado a la lengua inglesa del verso sanjuanista se encuentra en The collected works of St. Jolm of the Cross, translated by Kieran Kavanaugh $O C D$ and Otilio Rodriguez $O C D$, with introductions by Kieran Kavanaugh, Washington DC, Institute of Carmelite Studies, ICS Publications, 1979.

${ }^{15}$ Johannes vom Krelı. Geistlicher Gesang, Aus dem spanischen Übersetz von P. Aloysius ab Inmac. Conceptione, München, Wissenschaftliche Buchgesellschaft, Darmstadt, 1987.

I" Proponemos aqui también la versión que Paola Elia ofrece en Juan de la Cruz. Poesie, Roma, Japadre editore L'Aquila, 1988, y que la profesora toma de la edición de Venecia de las obras de San Juan de la Cruz de 1747: "un non sò che, che restan balbutiendo".
} 


\begin{tabular}{|l|l|}
\hline Italiano & Un no sé qué que dicen balbuciendo \\
\hline Francés & Un no sé qué que ellos quedan balbuciendo \\
\hline Inglés & De, ah!, yo-no-sé-qué detrás de sus balbuceos \\
\hline Alemán & $\begin{array}{l}\text { Esto (...que) ellos repiten sólo balbuceando a duras } \\
\text { penas }\end{array}$ \\
\hline
\end{tabular}

Estudiemos ahora estas traducciones desde diferentes puntos de vista. Respecto a la estructura métrica sólo conviene señalar que las traducciones italiana ${ }^{17}$ y francesa conservan el endecasílabo de la lengua original, mientras que las traducciones inglesa y alemana lo superan en cuanto a sílabas.

En cuanto a la estructura versal de las cuatro traducciones, diremos que es semejante a la estructura versal en la lengua original. Suponemos que los diferentes traductores, debido a la indudable fuerza expresiva del verso, han decidido conservar la misma estructura para no correr el riesgo de que se pierda dicha fuerza al mezclarlo con parte del resto de las estructuras versales de la lira. El que hemos denominado "elemento determinante" sólo se mantiene, aunque con pérdida de uno de los fonemas $/ \mathrm{k} /$, en las traducciones italiana y francesa. En el primer caso porque el verbo comienza con el fonema /d/, y en el segundo caso porque el verbo comienza con el fonema $/ \mathrm{r} /$. Por lo demás, estas traducciones mantienen casi intactas las unidades de sujeto y de verbo $y$, en este sentido, poco o nada varían respecto del verso original. Tenemos que resaltar, no obstante, la pérdida de la última vocal que se produce en el verbo "dire" de la traducción italiana. La forma ortográfica correcta es "(essi) dicono", pero el traductor ha optado por suprimir la "o" desinencial, lo que, por otra parte no influye en su comprensión ya que el presente de indicativo no admite en ninguna de sus seis personas una forma similar. Una alternativa al verbo "dire", que también es usada en la traducción italiana, es "andare", cuya tercera persona en plural del presente de indicativo es "vanno". Así el verso quedaría de la siguiente forma:

\section{un non so che che vanno balbettando}

Tal forma es comprensible también es lengua española, aunque en un registro de lenguaje más coloquial. Por ejemplo: "no sé qué andan (van) diciendo de mí..."

Diríamos, pues, que el traslado al italiano y al francés resulta menos conceptual y más literal que en las traducciones inglesa y alemana.

\footnotetext{
${ }^{17}$ De hecho, la traducción a la lengua italiana se abstiene de usar el verbo "rimanere", que en su tercera persona del plural del presente de indicativo es "rimangono" porque, además de carecer del carácter estético del utilizado, se saldría de la estructura métrica del verso en su lengua original.
} 
Otro asunto bien distinto lo configuran las traducciones inglesa y alemana que, como antes dijimos, por su peculiar estructura lingüistica, dan más prioridad al contenido, al mensaje, que a la forma del verso, cuestión que además tiene que ver estrechamente con la "teoría poética" de cada traductor y, por tanto, con la prioridad que otorga a la cuestión estética o a la cuestión expresiva ${ }^{18}$.

La traducción inglesa es quizás la menos estética y expresiva de las cuatro propuestas:

\section{Of, ah, I-don't-know-what behind their stammering}

Es cierto que, para el lector, conserva la unión entre guiones del sintagma "no sé qué" (I-don't-know-what) para que éste no pierda la estela del elemento que determina todo el verso. Se mantiene, igualmente, el verbo en la forma de gerundio, aunque, como vimos más arriba, la traducción al español lo convierta en un sustantivo. Quizás, y ahí dejamos la propuesta, una forma más estética de traducirlo y que conserva el mensaje sanjuanista hubiera sido:

\section{An "I-don't-know-what" they keep mumbling on}

La traducción alemana, por su parte, es, desde nuestro punto de vista, la más interesante de las cuatro, a pesar de que el traductor ha hecho más hincapié en la lengua traducida que en la lengua original del verso. Lo esencial de esta traducción consiste en que se ha realizado un considerable esfuerzo para ganar en las funciones expresiva y, sobre todo, comunicativa, a través del contenido, del mensaje. Por otra parte, por cuestiones evidentes, no se ha podido mantener el mismo nivel de literalidad que en las otras. De hecho, dos traducciones más convencionales, más literales, del verso hubieran sido las que siguen:

\section{Ein etwas, das $\mathrm{hr}$. Stammeln nicht kann geben o Ein "ich-wei $\beta$-nicht-was", das sie unablässig stammeln}

El traductor, como decimos, ha optado por una traducción distinta. Veamos en qué ha consistido este proceso.

\begin{tabular}{|l|l|}
\hline $\begin{array}{l}\text { Un no sé qué que quedan } \\
\text { balbuciendo }\end{array}$ & $\begin{array}{l}\text { Das sie nur mühsam stammelnd } \\
\text { wiedergeben }\end{array}$ \\
\hline
\end{tabular}

\footnotetext{
${ }^{18}$ Cfr. Newmark, ob. cit., p. 165.
} 
El traductor es consciente de que en esta lengua no puede conseguir el efecto sonoro del balbuceo o tartamudeo "que...que...que..." y, por tanto, trata de buscarlo con otros recursos que están a su alcance. Analicemos el verso en alemán:

\section{Das sie nur mühsam stammelnd wiedergeben}

Escribamos de nuevo, la traducción literal del verso en español:

Esto(...que) ellos repiten sólo balbuceando a duras penas

Comparémosla seguidamente con la de la lengua original:

Un no sé qué que quedan balbuciendo / esto (...que) ellos repiten sólo balbuceando a duras penas

El traductor del verso ha optado, como decimos, por una traducción "comunicativa" (según la terminología de Newmark) en la que el énfasis se pone sobre la lengua a la que se traduce el verso sanjuanista, más que por una traducción literal o una traducción palabra por palabra que, posiblemente, no funcionaría para la lengua alemana. Sin embargo, no por ello ha esquivado el esfuerzo por trasladar el contenido del mensaje del verso de San Juan de la Cruz a los lectores germanos. En primer lugar, y como ya dijimos anteriormente, aunque se conserva la estructura versal, se quiebra, no obstante, la estructura métrica al añadir el traductor más unidades léxicas al verso: dos adverbios que no aparecen en la lengua original como son 'nur' (sólo) y 'mühsam' (a duras penas, que puede funcionar también como adjetivo = penoso, fatigoso, laborioso). El verbo 'quedar', por otra parte, es sustituido por el verbo 'wiedergeben' (repetir). Este recurso expresivo, que se convierte en el elemento determinante del verso alemán, intenta salvar, y creemos que lo consigue, el efecto sonoro del 'que...que...que...' sanjuanista. Si en la lengua alemana no existe este efecto, es lógico que el traductor haya cambiado el verbo original por uno que pueda acercarse, al menos conceptualmente, a dicho efecto. El verbo 'balbucear' se mantiene usando el término alemán 'stammelnd' que, en esta forma de Partizip eins, conserva el mismo valor de gerundio que en la lengua de origen. El adverbio 'mühsam', en este caso, tiene la función de reforzar semánticamente el verbo, lo que en lengua española es innecesario porque ya se entiende que 'balbucear', término que indica el hablar de los niños (tal y como recogimos en el comentario que el mismo fraile Juan de la Cruz escribe de su verso), es una acción laboriosa y penosa. Ya lo hicimos notar también con anterioridad al recoger la definición 
que de este verbo realiza el diccionario de la Real Academia. El resultado final es como sigue:

Un no sé qué que. (ellos) quedan das / nur/ wiedergeben

Balbuciendo sie $/ \varnothing$ stammelnd / mühsam

Desde luego, la traducción alemana apenas conserva la literalidad del verso original español, pero no cabe duda de que el esfuerzo del traductor la ha convertido en una recreación casi perfecta del mensaje, del contenido o de la intención que San Juan de la Cruz quiso transmitir con la irrepetible musicalidad de este verso.

\section{Conclusión}

Como acabamos de ver, de las cuatro propuestas de traducción del verso de San Juan de la Cruz, dos de ellas, la francesa y la italiana, por evidente semejanza en las raíces lingüísticas y culturales, son prácticamente literales y conservan, por ello, el énfasis de la lengua original; las otras dos, en cambio, también por evidentes modificaciones en las raíces culturales y en la lengua, no han tenido más remedio que desprenderse de la literalidad del verso original tratando de buscar otros recursos que, de igual manera, satisfagan, por un lado, las intenciones del autor y, por otro, el acercamiento al lector. De las dos, sólo la traducción alemana ha conseguido este doble objetivo: respetar la intención de San Juan de la Cruz y conseguir que el lector se acerque lo máximo posible a los efectos comunicativos del verso, es decir, atender con igual intensidad tanto a la forma como al contenido del mensaje de la lengua original. 